\title{
HOMOTOPY GROUPS OF JOINS AND UNIONS
}

BY

\section{GEORGE W. WHITEHEAD}

1. Introduction. The homology groups of the join $X * Y$ of two spaces have long been known to satisfy a "Künneth formula." On the other hand, little is known about the homotopy groups of $X * Y$. There is a bilinear pairing $(\alpha, \beta) \rightarrow \alpha * \beta$ of $\pi_{p}(X)$ with $\pi_{q}(Y)$ to $\pi_{p+q+1}(X * Y) ; \alpha * \beta$ is called the join of $\alpha$ and $\beta$. If $Y=S^{n}$ is an $n$-sphere and $\iota$ generates $\pi_{n}(Y)$, then it follows from the Freudenthal suspension theorem that the map $\alpha \rightarrow \alpha * \iota$ is, for small $p$, an isomorphism of $\pi_{p}(X)$ with $\pi_{p+n+1}(X * Y)$. Thus, in low dimensions, the homotopy groups of $X * S^{n}$ are generated by the joins of the homotopy groups of the factors.

In this paper we consider the homotopy groups of the join of $X$ with an arbitrary 1 -connected $\mathrm{CW}$-complex $Y$. There is a spectral sequence whose initial term is, in low dimensions, the homology group $\left.{ }^{1}\right)$ of $Y$ with coefficients in the homotopy group $\left(^{1}\right)$ of $X$ and whose final term is the graded group of the homotopy group of $X * Y$ with respect to a suitable filtration. As a consequence, the homotopy groups of $X * Y$, even in low dimensions, are not, in general, generated by the joins of the homotopy groups of the factors. A further consequence is a new proof of the symmetry of the stable EilenbergMacLane groups, which was first proved by H. Cartan [4].

These results can be used to study the homotopy groups of the union $X \vee Y$ of two spaces $X$ and $Y$ with a single point in common. The space $X \vee Y$ can be naturally imbedded in $X \times Y$ and we have a natural isomorphism

$$
\pi_{n}(X \vee Y) \approx \pi_{n}(X) \oplus \pi_{n}(Y) \oplus \pi_{n+1}(X \times Y, X \vee Y)
$$

Now there is a homomorphism of $\pi_{n+1}(X \times Y, X \vee Y)$ into $\pi_{n+2}(X * Y)$, which is an isomorphism in low dimensions. Thus we can apply the above results on the homotopy groups of $X * Y$. In particular, the group $\pi_{n+1}(X \times Y, X \vee Y)$ is not in general generated by "generalized Whitehead products" of elements of the homotopy groups of $X$ and $Y$, even in low dimensions.

In a recent paper [0] M. G. Barratt and J. H. C. Whitehead have introduced an exact couple for an arbitrary $\mathrm{CW}$-triad. This exact couple, in the special case of the triad $(X \vee Y ; X, Y)$, seems to be closely related to the one we have introduced here. However, our results do not seem to follow

Presented to the Society, June 19, 1954 ; received by the editors August 4, 1955.

(1) By the homotopy (homology) group of a space $X$, we mean the direct sum of the homotopy (homology) groups in all dimensions. 
immediately from their work; furthermore we do not assume that $X$ is a CW-complex.

2. Joins. Let $X$ and $Y$ be spaces, which we assume to be disjoint from each other and from $X \times Y \times I$, where $I$ is the unit interval $\{t \mid 0 \leqq t \leqq 1\}$. Let $W=X \cup(X \times Y \times I) \cup Y$; we topologize $W$ by defining a subset to be open if and only if its intersection with each of the spaces $X, X \times Y \times I$, and $Y$ is open. The join of $X$ and $Y$ is the identification space $X * Y$ obtained from $W$ by identifying each $x \in X$ with all of the points $(x, y, 0)$ and each $y \in Y$ with all of the points $(x, y, 1)$. The identification map sends $X$ and $Y$ homeomorphically into $X * Y$; hence we may consider $X$ and $Y$ as subspaces of $X * Y$. Let $(1-t) x \oplus t y$ be the image of $(x, y, t)$ in $X * Y$.

The join operation is easily seen to be commutative (up to a natural homeomorphism). The join of $X$ with the empty set $\varnothing$ is $X$.

Let $f: X \rightarrow X^{\prime}, g: Y \rightarrow Y^{\prime}$ be maps. The join of $f$ and $g$ is the map

$$
f * g: X * Y \rightarrow X^{\prime} * Y^{\prime}
$$

defined by

$$
\begin{aligned}
(f * g)((1-t) x \oplus t y) & =(1-t) f(x) \oplus t g(y), \\
(f * g)(x) & =f(x), \quad(f * g)(y)=g(y) ;
\end{aligned}
$$

continuity of $f * g$ follows from the fact that the join has the identification topology. Since $X * Y$ is an identification space of $W$ and since $I$ is compact, it follows [18, Lemma 4] that $(X * Y) \times I$ is an identification space of $W \times I$. Hence, if $f, f^{\prime}: X \rightarrow X^{\prime}$ are homotopic and if $g, g^{\prime}: Y \rightarrow Y^{\prime}$ are homotopic, then $f * g$ and $f^{\prime} * g^{\prime}$ are homotopic maps of $X * Y$ into $X^{\prime} * Y^{\prime}$. It follows that, if $X$ and $X^{\prime}$ have the same homotopy type, and if $Y$ and $Y^{\prime}$ have the same homotopy type, then $X * Y$ and $X^{\prime} * Y^{\prime}$ have the same homotopy type.

If $f: X \subset X^{\prime}$ and $g: Y \subset Y^{\prime}$, then $f * g$ is a $1: 1$ map of $X * Y$ into $X^{\prime} * Y^{\prime}$; if furthermore $X$ is closed in $X^{\prime}$ and $Y$ is closed in $Y^{\prime}$, then $f * g$ is a homeomorphism and its image is closed in $X^{\prime} * Y^{\prime}$; in that case we may consider $X * Y$ as a closed subset of $X^{\prime} * Y^{\prime}$.

Note that the join of the inclusion map of $\varnothing$ into $X^{\prime}$ with any map $g: Y \rightarrow Y^{\prime}$ is the composition of $g$ with the inclusion map of $Y^{\prime}$ into $X^{\prime} * Y^{\prime}$.

Let $P$ be a fixed space consisting of a single point $p$. The cone over $X$ is the join $\widehat{X}=X * P$. Let $A$ be a closed subspace of $X$; then the quotient space of $X$ by $A$ is the subspace $X \div A=X \cup \widehat{A}$ of $\widehat{X}$. The following properties are easily verified:

(2.1) $\widehat{X}$ is contractible;

(2.2) The triad $(X \div A ; X, \widehat{A})$ is proper.

Let $P^{\prime}$ be a fixed space consisting of two points $p_{+}, p_{-}$. The suspension $S(X)$ of $X$ is the join $X * P^{\prime}$. Note that $S(X)=\widehat{X}_{+} \cup \widehat{X}_{-}$, where $\widehat{X}_{ \pm}=X * p_{ \pm}$, while $\widehat{X}_{+} \cap \widehat{X}_{-}=X$. Thus $S(X)$ is the union of two contractible spaces whose intersection is $X$. It is easy to see that the triad $\left(S(X) ; \widehat{X}_{+}, \widehat{X}_{-}\right)$is proper. 
Let $\sigma, \tau$ be ordered Euclidean simplexes with vertices $a_{0}<\cdots<a_{p}$, $b_{0}<\cdots<b_{q}$ respectively. Then $\sigma * \tau$ has a natural affine structure, and we may regard $\sigma * \tau$ as an ordered Euclidean simplex with vertices $a_{0}<\cdots<a_{p}$ $<b_{0}<\cdots<b_{q}$. We consider the empty set as an ordered Euclidean ( -1$)-$ simplex. With the usual definition of equivalence of singular simplexes, we define $\widetilde{S}_{p}(X)$ to be the free abelian group generated by the singular $(p-1)$ simplexes in $X(p=0,1,2, \cdots)$ and define $\tilde{S}(X)=\sum_{p=0}^{\infty} \widetilde{S}_{p}(X)$, with the usual boundary operator (the boundary of each singular 0 -simplex is the unique singular (-1)-simplex), to be the augmental total singular complex of $X$. If $A \subset X$, then $\tilde{S}(A)$ is a subcomplex of $\tilde{S}(X)$ and we define $\widetilde{H}_{p}(X, A)$ to be the $(p+1)$ st homology group of the complex $\tilde{S}(X) / \widetilde{S}(A)$. Of course, $\tilde{H}_{p}(X, A)$ $=H_{p}(X, A)(p \geqq 0)$, except that $\tilde{H}_{0}(X, \varnothing)$ is the reduced 0-dimensional homology group of $X$.

Let $\phi: \sigma \rightarrow X, \psi: \tau \rightarrow Y$ be singular simplexes; then $\phi * \psi: \sigma * \tau \rightarrow X * Y$ is a singular simplex. The map $\phi \otimes \psi \rightarrow \phi * \psi$ induces a homomorphism $\alpha: \tilde{S}(X)$ $\otimes \widetilde{S}(Y) \rightarrow \widetilde{S}(X * Y)$, which is easily seen to be a chain mapping. It is known that $\alpha$ is, in fact, a chain-equivalence $\left({ }^{2}\right)$. Furthermore, $\alpha$ is natural in the sense that, if $f: X \rightarrow X^{\prime}$ and $g: Y \rightarrow Y^{\prime}$ are maps, and if $f^{\prime}, g^{\prime},(f * g)^{\prime}$ are the chain maps induced by $f, g$, and $f * g$, then the diagram

$$
\begin{array}{cc}
\tilde{S}(X) \otimes \tilde{S}(Y) \stackrel{f^{\prime} \otimes g^{\prime}}{\longrightarrow} & \tilde{S}\left(X^{\prime}\right) \otimes \tilde{S}\left(Y^{\prime}\right) \\
& \downarrow \alpha \quad 1 \alpha \\
\tilde{S}(X * Y) \stackrel{(f * g)^{\prime}}{\longrightarrow} & \tilde{S}\left(X^{\prime} * Y^{\prime}\right)
\end{array}
$$

is commutative. Hence, if $A$ is a closed subset of $Y, \alpha$ induces a chain-equivalence between the complexes $\tilde{S}(X) \otimes[\tilde{S}(Y) / \tilde{S}(A)]$ and $\tilde{S}(X * Y) / \tilde{S}(X * A)$. Hence

(2.3) The singular homology groups of $(X * Y, X * A)$ are given by $\tilde{H}_{q+1}(X * Y, X * A) \approx \sum_{i+j=q} \tilde{H}_{i}(X) \otimes \widetilde{H}_{j}(Y, A) \oplus \sum_{i+j=q-1} \operatorname{Tor}\left\{\tilde{H}_{i}(X), \tilde{H}_{j}(Y, A)\right\}$.

It is also known [13, Lemma 2.2$]$ that

(2.4) If $X$ is 0 -connected and $Y \neq \varnothing$, then $X * Y$ is 1-connected.

Consequently we can conclude from the relative Hurewicz theorem $[1,(23.3)]$ :

(2.5) If $X$ is $(m-1)$-connected $(m \geqq 1),(Y, A)$ is $(n-1)$-connected $(n \geqq 2)$, and $A$ is 1-connected, then $(X * Y, X * A)$ is $(m+n)$-connected, and

$$
\pi_{m+n+1}(X * Y, X * A) \approx \pi_{m}(X) \otimes \pi_{n}(Y, A) .
$$

The group $\tilde{H}_{p}(X) \otimes \widetilde{H}_{q}(Y, A)$ has a natural imbedding in $H_{p+q+2}(S(X)$

(2) This fact does not seem to be stated explicitly in the literature, but is not difficult to deduce from Milnor's proof [13] of the "Künneth theorem" for the homology groups of the join. The author is indebted to Milnor for the opportunity of reading his manuscript. 
$\otimes[S(Y) / S(A)])$; hence $\alpha$ induces a monomorphism of $\tilde{H}_{p}(X) \otimes \tilde{H}_{q}(Y, A)$ into $\tilde{H}_{p+q+1}(X * Y, X * A)$. If $u \in H_{p}(X), v \in H_{q}(Y, A)$, we define $u * v$ to be the image of $u \otimes v$ under the monomorphism.

Let $(S, a, \sigma)$ be an oriented $p$-sphere (i.e., $S$ is a $p$-sphere, $a \in S$, and $\sigma$ is a generator of $\left.\tilde{H}_{p}(S)\right)$ and let $(E, b, \epsilon)$ be an oriented $q$-cell (i.e. $E$ is a $q$-cell, $b$ is a point of the boundary $\dot{E}$ of $E$, and $\epsilon$ is a generator of $\left.\tilde{H}_{q}(E, \dot{E})\right)$. It is well known that $S * E$ is a $(p+q+1)$-cell with boundary $S * \dot{E}$; it follows from (2.1) that $\sigma * \epsilon$ is a generator of $H_{p+q+1}(S * E, S * \dot{E})$. Let $c=a / 2 \oplus b / 2$, then $c \in S * \dot{E}$ and $(S * E, c, \sigma * \epsilon)$ is an oriented $(p+q+1)$-cell. Let $X, Y$ be spaces, $A$ a closed subspace of $Y, x_{0} \in X, y_{0} \in A$, and let $z_{0}=x_{0} / 2 \oplus y_{0} / 2 \in X * Y$. Then if $f:(S, a) \rightarrow\left(X, x_{0}\right), g:(E, \dot{E}, b) \rightarrow\left(Y, A, y_{0}\right)$, the homotopy class of $f * g:(S * E, S * \dot{E}, c) \rightarrow\left(X * Y, X * A, z_{0}\right)$ depends only on the homotopy classes of the maps $f, g$; thus the correspondence $(f, g) \rightarrow f * g$ induces a pairing of $\pi_{p}(X)$ with $\pi_{q}(Y, A)$ to $\pi_{p+q+1}(X * Y, X * A)$. If $\alpha, \beta$ are the homotopy classes of $f, g$ we define $\alpha * \beta$ to be the homotopy class of $f * g$.

(2.6) The operation $(\alpha, \beta) \rightarrow \alpha * \beta$ is bilinear.

The proof is similar to that of (3.20) in [17].

(2.7) Let $f:\left(X, x_{0}\right) \rightarrow\left(X^{\prime}, x_{0}^{\prime}\right)$ and $g:\left(Y, A, y_{0}\right) \rightarrow\left(Y^{\prime}, A^{\prime}, y_{0}^{\prime}\right)$ be maps. Then for any $\alpha \in \pi_{p}(X), \beta \in \pi_{q}(Y, A)$, we have

$$
(f * g)_{*}(\alpha * \beta)=f_{*}(\alpha) * g_{*}(\beta) .
$$

The proof is trivial.

(2.8) Let $X$ be a space, $(Y, A, B)$ a triple, and let $x_{0} \in X, y_{0} \in B$. Let $\alpha \in \pi_{p}(X), \beta \in \pi_{q}(Y, A)$. Let $\partial: \pi_{q}(Y, A) \rightarrow \pi_{q-1}(A, B)$ and $\partial^{\prime}: \pi_{p+q+1}(X * Y$, $X * A) \rightarrow \pi_{p+q}(X * A, X * B)$ be the homotopy boundary operators of the appropriate triples. Then

$$
\partial^{\prime}(\alpha * \beta)=(-1)^{p+1} \alpha * \partial \beta .
$$

The proof of this fact is routine, and is left to the reader.

3 . The spaces $L(\Pi, n)$. Let $\Pi$ be an abelian group, $n$ an integer $\geqq 2$. Then there is a $\mathrm{CW}$-complex $L$ such that

(1) $L$ is 1 -connected;

(2) $\tilde{H}_{q}(L)=0$ for $q \neq n$;

(3) $\pi_{n}(L) \approx \Pi$.

Any two such complexes have the same homotopy type. By $L(\Pi, n)$ we shall mean a $\mathrm{CW}$-complex $L$ satisfying the above conditions, together with a fixed isomorphism between $\pi_{n}(L)$ and $\Pi$. We may therefore identify the (isomorphic) groups $\pi_{n}(L)$ and $H_{n}(L)$ with $\Pi$. If $\phi: \Pi \rightarrow G$ is a homomorphism, then there is a map $f: L(\Pi, n) \rightarrow L(G, n)$ such that the induced homomorphism $f_{*}: H_{n}(L(\Pi, n)) \rightarrow H_{n}(L(G, n c)$ is equal to $\phi$. However, the homotopy class of $f$ may not be uniquely determined by $\phi$; it is unique if $\Pi$ and $G$ are free. If II is free, we may take $L(\Pi, n)$ to be a cluster of spheres; in particular, we may take $L(Z, n)=S^{n}$. 
The above facts follow from the work of Eilenberg $[5 ; 6]$; the spaces $L(\Pi, n)$ have been studied by Moore [15] and more recently by Peterson [16].

TheOREM 3.1. Let $X$ be an ( $m-1)$-connected space $(m \geqq 2)$, $\Pi$ a free abelian group, $n \geqq 2$. Then the homomorphism

$$
\phi: \pi_{q}(X) \otimes \Pi \rightarrow \pi_{q+n+1}(X * L(\Pi, n)),
$$

induced by the join operation is an isomorphism for $q \leqq 2 m-2$, and an epimorphism for $q=2 m-1$.

Proof. We may assume the $L=L(\Pi, n)$ is a cluster of $n$-spheres; i.e., $L=\bigcup_{\alpha} S_{\alpha}$, where each $S_{\alpha}$ is an $n$-sphere and $\alpha \neq \beta$ implies $S_{\alpha} \cap S_{\beta}=\bigcap_{\gamma} S_{\gamma}$ is a single point $\dot{y}_{0}$. If $\Pi$ has rank 1 , then $X * L$ is the $(n+1)$-fold suspension of $X$ and the theorem follows by iteration from a known generalization of the Freudenthal suspension theorem $\left({ }^{3}\right)$. Suppose that $\Pi$ has finite rank; then $\left(X * S_{\alpha}\right) \cap\left(X * S_{\beta}\right)$ is the contractible set $X * y_{0}$; since $X * S_{\alpha}$ is $(m+n)$-connected, our result follows immediately by induction from the statement:

(3.2) Let $C=A \cup B$, where $A$ and $B$ are closed in $C, A \cap B$ is contractible, and $A \cap B$ is a strong neighborhood deformation retract of one of the sets $A, B$. Suppose that $A$ is r-connected, $B$ is s-connected. Then, for $q \leqq r+s-1$, the injections induce an isomorphism: $\pi_{q}(A) \oplus \pi_{q}(B) \approx \pi_{q}(C)$.

Proof of (3.2). By exactness of the homotopy sequence

$$
\cdots \rightarrow \pi_{q}(A \cap B) \rightarrow \pi_{q}(A) \oplus \pi_{q}(B) \rightarrow \pi_{q}(A / B) \rightarrow \pi_{q-1}(A \cap B) \rightarrow \cdots
$$

of the covering $(A / B)[11]$, we have

$$
\pi_{q}(A) \oplus \pi_{q}(B) \approx \pi_{q}(A / B)
$$

for all $q$. Furthermore, the triad $(C ; A, B)$ is $(r+s)$-connected, since $(A, A \cap B)$ is $r$-connected and $(B, A \cap B)$ is $s$-connected [3]. From exactness of the sequence [11]

$$
\cdots \rightarrow \pi_{q+1}(C ; A, B) \rightarrow \pi_{q}(A / B) \rightarrow \pi_{q}(C) \rightarrow \pi_{q}(C ; A, B) \rightarrow \cdots
$$

we conclude

$$
\pi_{q}(A / B) \approx \pi_{q}(C)
$$

for $q \leqq r+s-1$. The truth of (3.2) now follows from the fact that the composition of the homomorphisms in (1) and (2) is induced by the injections.

The truth of Theorem 3.1 in the general case now follows by standard arguments once we have proved

(3.3) Let $X$ be a space, $L$ a $C W$-complex, $K$ a compact subset of $X * L$. Then there is a finite subcomplex $L_{1}$ of $L$ such that $K \subset X * L_{1}$.

(3) This is an immediate consequence of a theorem of Blakers and Massey on the homotopy groups of a triad [3, Theorem I]. 
Proof of (3.3). Define a map $f: X * L \rightarrow \widehat{X} \times \widehat{L}$ by

$$
f((1-t) x \oplus t y)= \begin{cases}(x,(1-2 t) p \oplus 2 t y) & (0 \leqq t \leqq 1 / 2), \\ (2-2 t) x \oplus(1-2 t) p, y & (1 / 2 \leqq t \leqq 1) ;\end{cases}
$$

$f$ is well-defined, and therefore continuous, since $X * Y$ has the identification topology; furthermore, $f$ is $1: 1$. Let $\pi: \widehat{X} \times \widehat{L} \rightarrow \widehat{L}$ be the projection on the second factor. Then $\pi(f(K))$ is a compact subset of $\widehat{L}$, and $\widehat{L}$ is a CW-complex $[19,(\mathrm{~F})]$. Hence there is a finite subcomplex $L_{1}$ of $L$ such that $\pi(f(K)) \subset \widehat{L}_{1}$. Hence $f(K) \subset \widehat{X} \times \widehat{L}_{1}$, and it follows easily that $K \subset X * L_{1}$.

4. Homotopy resolutions of a pair. Let $(X, A)$ be a pair, $x_{0} \in A ; x_{0}$ will be the base point for all homotopy groups mentioned in this section. For simplicity, we assume that $(X, A)$ is 1-connected. A homotopy resolution of $(X, A)$ is a sequence $\left\{X_{n} \mid n=0, \pm 1, \pm 2, \cdots\right\}$ of subspaces of $X$ such that

(1) $A \subset X_{n} \subset X_{n+1}$ for all $n$;

(2) $\left(X_{n+1}, X_{n}\right)$ is 1 -connected;

(3) for each $q, \pi_{q}\left(X_{n}, A\right)=0$ for $n$ sufficiently small;

(4) for each $q, \pi_{q}\left(X, X_{n}\right)=0$ for $n$ sufficiently large.

Let $\left\{X_{n}\right\}$ be a homotopy resolution of $(X, A)$. We define an exact couple $[12]\langle D, E ; i, j, \partial\rangle$ as follows:

$$
\begin{aligned}
& D=\sum_{p, q} D_{p, q}, \quad D_{p, q}=\pi_{p+q}\left(X_{p}, A\right) \\
& E=\sum_{p, q} E_{p, q}, \quad E_{p, q}=\pi_{p+q}\left(X_{p}, X_{p-1}\right) \\
& \left.\begin{array}{r}
i \\
j
\end{array}\right\} \text { are bihomogeneous of degrees }\left\{\begin{array}{l}
(-1,0) \\
(1,-1) \\
(0,0) ;
\end{array}\right.
\end{aligned}
$$

(direct sums);

$$
\begin{aligned}
& \text { d: } \quad E_{p, q} \rightarrow D_{p-1, q} \mid \text { are the homomorphisms of the homotopy }
\end{aligned}
$$

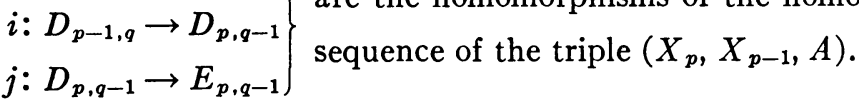

Let $\left\langle D^{k}, E^{k} ; i^{(k)}, j^{(k)}, \partial^{(k)}\right\rangle$ be the $(k-1)$ st derived couple.

Let $\pi_{p, q}$ be the image of the injection: $\pi_{p+q}\left(X_{p}, A\right) \rightarrow \pi_{p+q}(X, A)$, and define $\bar{\pi}(X, A)$ to be the direct sum $\sum_{p, q} \pi_{p, q} / \pi_{p-1, q+1}$.

THEOREM 4.1. For each $p, q$, we have $E_{p, q}^{k}=E_{p, q}^{k+1}$ for $k$ sufficiently large. Thus the direct limit $E_{p, q}^{\infty}=\lim _{k \rightarrow \infty} E_{p, q}^{k}$ exists, and

$$
E_{p, q}^{\infty} \approx \pi_{p, q} / \pi_{p-1, q+1} \text {. }
$$

Hence $E^{\infty}=\sum_{p, q} E_{p, q}^{\infty} \approx \bar{\pi}(X, A)$.

Proof. An easy computation shows that

$$
E^{k}=\partial^{-1}\left(\text { Image } i^{k-1}\right) / j\left(\text { Kernel } i^{k-1}\right),
$$


where $i^{k-1}$ is the $(k-1)$ st iterate of $i$ (and not the homomorphism $i^{(k-1)}$ of the $(k-2)$ nd derived couple). Thus

where

$$
E_{p, q}^{k}=U / V
$$

$U$ is the set of all $x \in E_{p, q}$ such that $\partial x$ belongs to the image of the injection: $\pi_{p+q-1}\left(X_{p-k}, A\right) \rightarrow \pi_{p+q-1}\left(X_{p-1}, A\right)$;

$V$ is the set of all elements of $E_{p, q}$ of the form $j y$, where $y$ belongs to the kernel of the injection: $\pi_{p+q}\left(X_{p}, A\right) \rightarrow \pi_{p+q}\left(X_{p+k-1}, A\right)$.

Now for $k$ sufficiently large, $\pi_{p+q-1}\left(X_{p-k}, A\right)=0$ and $\pi_{p+q+1}\left(X, X_{p+k-1}\right)=0$. Then the injection: $\pi_{p+q}\left(X_{p+k-1}, A\right) \rightarrow \pi_{p+q}(X, A)$ is a monomorphism, and therefore

$U$ is the set of all $x \in E_{p, q}$ such that $\partial x=0$;

$V$ is the set of all elements of $E_{p, q}$ of the form $j y$, where $y$ be'ongs to the kernel of the injection: $\pi_{p+q}\left(X_{p}, A\right) \rightarrow \pi_{p+q}(X, A)$.

Thus $U$ and $V$ are independent of $k$ if $k$ is sufficiently large. Hence $E_{p, q}^{k}=U / V$ is also.

To prove the second statement, consider the diagram

$$
\begin{gathered}
\pi_{p+q}\left(X_{p-1}, A\right) \\
\downarrow i \\
D_{p, q}=\pi_{p+q}\left(X_{p}, A\right) \stackrel{\jmath}{\rightarrow} \pi_{p+q}\left(X_{p}, X_{p-1}\right) \stackrel{\partial}{\rightarrow} \pi_{p+q-1}\left(X_{p-1}, A\right) \\
\downarrow i^{\prime} \\
\pi_{p+q}(X, A)
\end{gathered}
$$

Now, for sufficiently large $k, U=$ Kernel $\partial=$ Image $j \approx D_{p, q} /$ Kernel $j$, and $V=j\left(\right.$ Kernel $\left.i^{\prime}\right) \approx\left(\right.$ Kernel $i^{\prime}+$ Kernel $\left.j\right) /$ Kernel $j$; thus

$$
\begin{aligned}
E_{p, q}^{\infty}=E_{p, q}^{k} & =\frac{D_{p, q} / \text { Kernel } j}{\left(\text { Kernel } i^{\prime}+\text { Kernel } j\right) / \text { Kernel } j} \\
& \approx \frac{D_{p, q}}{\operatorname{Kernel} i^{\prime}+\text { Kernel } j} \\
& \approx-\frac{D_{p, q} / \text { Kernel } i^{\prime}}{\left(\text { Kernel } i^{\prime}+\text { Kernel } j\right) / \text { Kernel } i^{\prime}} \\
& =\frac{\text { Image } i^{\prime}}{i^{\prime}(\text { Kernel } j)}=\frac{\text { Image } i^{\prime}}{i^{\prime}(\text { Image } i)} \\
& =\frac{\text { Image } i^{\prime}}{\text { Image }\left(i^{\prime} \circ i\right)} \\
& =\frac{\pi_{p, q}}{\pi_{p-1 . q+1}} \cdot
\end{aligned}
$$


REMARK 1. For each $n, \pi_{p, n-p}=0$ for $p$ sufficiently small and $\pi_{p, n-p}$ $=\pi_{n}(X, A)$ for $p$ sufficiently large; thus the chain of subgroups $\left\{\pi_{p, q} \mid p+q\right.$ $=n\}$ of $\pi_{n}(X, A)$ is finite.

REMARK 2. We may define homology and cohomology resolutions in a similar way, and results analogous to the above hold. The above results constitute a trivial extension of Massey's exact couples [12], which are concerned with the resolution given by the skeleta of a triangulation of $(X, A)$.

5. The spectral sequence of a join. Let $X$ be an $(m-1)$-connected space, $Y$ an $(n-1)$-connected CW-complex $(m, n>1)$. Let $Y^{p}$ be the $p$-skeleton of $Y$; without loss of generality, as far as the homotopy type of $Y$ is concerned, we may assume that $Y^{n-1}$ is a single point $y_{0}$.

Lemma 5.1. The sequence $\left\{X * Y^{n+p} \mid p=0, \pm 1, \pm 2, \cdots\right\}$ forms a homotopy resolution of $\left(X * Y, X * y_{0}\right)$.

Proof. If $p<0, X * Y^{n+p}=X * y_{0}$ is contractible. On the other hand, if $p \geqq 0,\left(Y, Y^{n+p}\right)$ is $(n+p)$-connected and hence, by (2.6), $\left(X * Y, X * Y^{n+p}\right)$ is $(m+n+p-1)$-connected, so that $\pi_{q}\left(X * Y, X * Y^{n+p}\right)=0$ for $p \geqq q-m-n-1$.

For convenience, we re-index the terms of the spectral sequence of the above homotopy resolution of $\left(X * Y, X * y_{0}\right)$ by setting

thus

$$
\begin{aligned}
& E_{p, q}^{r}=E_{p, m+q+1}^{r}, \\
& \pi_{p, q}=\pi_{p, m+q+1} ;
\end{aligned}
$$

$$
\begin{aligned}
E_{p, q}^{1} & =\pi_{m+n+p+q+1}\left(X * Y^{n+p}, X * Y^{n+p-1}\right), \\
E_{p, q}^{r} & =0 \text { if } p<0 \text { or } q<0 ; \\
\pi_{m+n+s+1}(X * Y) & =\pi_{s, 0} \supset \pi_{s-1,1} \supset \cdots \supset \pi_{0, s} \supset \pi_{-1, s+1}=0, \\
\pi_{p, q} / \pi_{p-1, q+1} & =E_{p, q}^{\infty}
\end{aligned}
$$

We identify $\pi_{n+p}\left(Y^{n+p}, Y^{n+p-1}\right)$ with $C_{n+p}(Y)$. Then the join operation defines a homomorphism

$$
\phi: \pi_{m+q}(X) \otimes C_{n+p}(Y) \rightarrow \pi_{m+n+p+q+1}\left(X * Y^{n+p}, X * Y^{n+p-1}\right)=E_{p, q}^{1} .
$$

LemMa 5.2. The homomorphism

$$
\phi: \pi_{m+q}(X) \otimes C_{n+p}(Y) \rightarrow E_{p, q}^{1}
$$

is an isomorphism for $q \leqq m-2$ and an epimorphism for $q=m-1$.

Proof. Note that $Y^{n+p} \div Y^{n+p-1}=L\left(C_{n+p}(Y), n+p\right)$, the isomorphism $C_{n+p}(Y) \approx H_{n+p}\left(Y^{n+p} \div Y^{n+p-1}\right)$ being $\beta^{-1} \circ \alpha$, where $\pi_{n+p}\left(Y^{n+p}, Y^{n+p-1}\right) \stackrel{\alpha}{\rightarrow} \pi_{n+p}\left(Y^{n+p} \div Y^{n+p-1},\left(Y^{n+p-1}\right) \frown\right) \stackrel{\beta}{\rightarrow} \pi_{n+p}\left(Y^{n+p} \div Y^{n+p-1}\right) ;$ $\alpha$ and $\beta$ are injections. 
Consider the diagram

$$
\begin{aligned}
& \pi_{m+q}(X) \otimes \pi_{n+p}\left(Y^{n+p}, Y^{n+p-1}\right) \stackrel{\phi}{\rightarrow} \pi_{m+n+p+q+1}\left(X * Y^{n+p}, X * Y^{n+p-1}\right) \\
& \downarrow 1 \otimes \alpha \\
& \pi_{m+q}(X) \otimes \pi_{n+p}\left(Y^{n+p} \div Y^{n+p-1},\left(Y^{n+p-1}\right) \multimap \stackrel{\phi^{\prime}}{\rightarrow} \pi_{m+n+p+q+1}\left(X *\left(Y^{n+p} \div Y^{n+p-1}\right), X *\left(Y^{n+p-1}\right)-\right)\right. \\
& \uparrow 1 \otimes \beta \\
& \pi_{m+q}(X) \otimes \pi_{n+p}\left(Y^{n+p} \div Y^{n+p-1}\right) \stackrel{\phi^{\prime \prime}}{\rightarrow} \pi_{m+n+p+q+1}\left(X *\left(Y^{n+p} \div Y^{n+p-1}\right)\right)
\end{aligned}
$$

where $i, i^{\prime}$ are injections, and $\phi^{\prime}, \phi^{\prime \prime}$ are also induced by the join operation. Because of (2.7), the diagram is commutative.

Now $i$ is an excision, and the triad $\left(X *\left(Y^{n+p} \div Y^{n+p-1}\right) ; X * Y^{n+p}\right.$, $X *\left(Y^{n+p+1}\right)$ ) $)$ is proper. Furthermore $\left(X * Y^{n+p}, X * Y^{n+p-1}\right)$ is $(m+n+p)$ connected, by (2.5), since $X$ is $(m-1)$-connected and $\left(Y^{n+p}, Y^{n+p-1}\right)$ is $(n+p-1)$-connected. Also $\left(X *\left(Y^{n+p-1}\right) \uparrow, X * Y^{n+p-1}\right)$ is $(m+n+1)$-connected, again by $(2.5)$, since $X$ is $(m-1)$-connected and $\left(\left(Y^{n+p-1}\right) \uparrow, Y^{n+p-1}\right)$ is $n$-connected. Hence the above triad is $(2 m+2 n+p+1)$-connected [ 3 ], and therefore $i$ is an isomorphism provided that $m+n+p+q+2 \leqq 2 m+2 n+p+1$, i.e. $q \leqq m+n-1$.

On the other hand, by Theorem $3.1, \phi^{\prime \prime}$ is an isomorphism for $q \leqq m-2$ and an epimorphism for $q=m-1$. Since $1 \otimes \alpha, 1 \otimes \beta$, and $i^{\prime}$ are always isomorphisms, our conclusion follows from the commutativity of the diagram.

LEMмA 5.3. Under the isomorphism of Lemma 5.2, the boundary operator $d_{1}$ of the spectral sequence $\left\{E^{r}\right\}$ is transformed into the operator $\partial_{1}$ given by

$$
\partial_{1}(\alpha \otimes \beta)=(-1)^{m+q+1} \alpha \otimes \partial \beta
$$

for $\alpha \in \pi_{m+q}(X), \beta \in C_{n+p}(Y), q \leqq m-2$.

Proof. By definition, $d_{1}$ is the homotopy boundary operator of the triple $\left(X * Y^{n+p}, X * Y^{n+p-1}, X * Y^{n+p-2}\right)$, while $\partial$ is the homotopy boundary operator of the triple $\left(Y^{n+p}, Y^{n+p-1}, Y^{n+p-2}\right)$. We then have

$$
\begin{aligned}
d_{1} \phi(\alpha \otimes \beta) & =d_{1}(\alpha * \beta) \\
& =(-1)^{m+q+1}(\alpha * \partial \beta) \\
& =\phi\left((-1)^{m+q+1} \alpha \otimes \partial \beta\right) .
\end{aligned}
$$

Corollary 5.4. For $q \leqq m-2, E_{p, q}^{2} \approx \tilde{H}_{n+p}\left(Y ; \pi_{m+q}(X)\right)$.

We summarize the results implied by the above discussion in

TheOREM 5.5. Let $X$ be an $(m-1)$-connected space, $Y$ an $(n-1)$-connected $C W$-complex $(m, n \geqq 2)$. Then there is a spectral sequence $\left\{E^{r}\right\}$ such that $E_{p, q}^{2} \approx \tilde{H}_{n+p}\left(Y ; \pi_{m+q}(X)\right)$ for $q \leqq m-2$ and $E^{\infty}$ is the graded group $\overline{\boldsymbol{x}}(X * Y)$ of $\sum_{r} \pi_{r}(X * Y)$ with respect to a suitable filtration.

6. The groups $A_{n}(\Pi ; G)$. Eilenberg and MacLane [7] have introduced the groups $H_{q}(\Pi, n ; G)$; they are the homology groups, with coefficients in $G$, of 
any space $K(\Pi, n)$ such that

$$
\begin{aligned}
& \pi_{q}(K(\Pi, n))=0 \\
& \pi_{n}(K(\Pi, n)) \approx \pi .
\end{aligned}
$$$$
(q \neq n)
$$

They have also shown [8] that the groups $H_{q}(\Pi, n ; G)$ satisfy a suspension theorem; there is a natural homomorphism $\sigma: H_{q}(\Pi, n ; G) \rightarrow H_{q+1}(\Pi, n+1 ; G)$ which is an isomorphism for $q \leqq 2 n-1$. Thus, for each $k$, the groups $H_{n+k}(\Pi, n ; G)$ are constant for $n$ sufficiently large; we denote this "stable" group by $A_{k}(\Pi ; G)$.

In his investigation of the Eilenberg-MacLane groups, H. Cartan [4] has shown that the groups $A_{k}(\Pi ; G)$ satisfy a "symmetry relation"

$$
A_{k}(\Pi ; G) \approx A_{k}(G ; \Pi) \text {. }
$$

We now show that this symmetry relation is a consequence of the results of $\$ 5$.

Theorem 6.1. Let $X=K(G, m)$ and let $Y$ be an $(n-1)$-connected $C W$ complex $(m, n \geqq 2)$. Then, for $s \leqq m-1$,

$$
\pi_{m+n+s+1}(X * Y) \approx \tilde{H}_{n+s}(Y ; G) .
$$

Proof. We have seen that, in the spectral sequence of $\$ 5$,

$$
E_{p, q}^{1} \approx \pi_{m+q}(X) \otimes C_{n+p}(Y) \quad(q \leqq m-2),
$$

while $E_{p, m-1}^{1}$ is a factor group of $\pi_{2 m-1}(X) \otimes C_{n+p}(Y)=0$. Thus, for $q \leqq m-1$,

$$
E_{q p,}^{2} \approx H_{n+p}\left(Y ; \pi_{m+q}(X)\right)= \begin{cases}0 & (q \neq 0), \\ H_{n+p}(Y ; G) & (q=0) .\end{cases}
$$

It follows that

$$
E_{p, q}^{r}=0 \quad(0 \neq q \leqq m-1 ; r=2,3, \cdots, \infty) .
$$

Now $d_{r}: E_{p, 0}^{r} \rightarrow E_{p-r, r-1}^{r}$ and $d_{r}: E_{p+r, 1-r}^{r} \rightarrow E_{p, 0}^{r}$. If $r \geqq 2$, then $E_{p+r, 1-r}^{r}=0$ since $1-r<0$; if $r>p$, then $E_{p-r, r-1}^{r}=0$ since $p-r<0$; finally, if $2 \leqq r \leqq p \leqq m$, then $0<r-1 \leqq p-1 \leqq m-1$ and hence $E_{p-r, r-1}^{r}=0$. Thus

$$
E_{p, 0}^{\infty} \approx E_{p, 0}^{2} \approx H_{n+p}(Y ; G) \quad(p \leqq m) .
$$

Now $\pi_{m+n+s+1}(X * Y)=\pi_{s, 0} \supset \pi_{s-1,1} \supset \cdots \supset \pi_{0, s} \supset \pi_{-1, s+1}=0$, and

$$
\pi_{p, q} / \pi_{p-1, q+1} \approx E_{p, q}^{\infty} .
$$

Thus, if $s \leqq m-1$, it follows from $\left(^{*}\right)$ that

$$
\pi_{s-1,1}=\cdots=\pi_{0, s}=0
$$

and therefore 


$$
\pi_{m+n+s+1}(X * Y)=\pi_{s, 0} \approx E_{s, 0}^{\infty} .
$$

Our result now follows from $\left({ }^{* *}\right)$.

Corollary 6.2. For any abelian groups $\Pi, G$ and any integer $s$,

$$
A_{s}(\Pi ; G) \approx A_{s}(G ; \Pi) .
$$

For we may take $m=n>s, Y=K(\Pi, n)$ above and conclude

$$
\begin{aligned}
& A_{s}(\Pi ; G)=H_{n+s}(\Pi, n ; G)=H_{n+s}(Y ; G) \\
& \quad \approx \pi_{m+n+s+1}(X * Y) \approx \pi_{m+n+s+1}(Y * X) \approx A_{s}(G ; \Pi)
\end{aligned}
$$

since $X * Y$ is homeomorphic with $Y * X$.

7. Homotopy groups of the union of two spaces with a point in common. Each space $X$ considered in this section will be 1 -connected and will have a distinguished base point $x_{0}$. In order to justify our use below of the Künneth theorem for homology groups of pairs, we assume that $\left\{x_{0}\right\}$ is a strong neighborhood deformation retract of $X$.

Let $X \vee Y$ be the subset $X \times y_{0} \cup x_{0} \times Y$ of $X \times Y ; X \vee Y$ is the union of the two spaces $X$ and $Y$ with the points $x_{0}$ and $y_{0}$ identified. Let $i_{1}: \pi_{n}(X)$ $\rightarrow \pi_{n}(X \bigvee Y), i_{2}: \pi_{n}(Y) \rightarrow \pi_{n}(X \bigvee Y)$ be injections, and let $\partial: \pi_{n+1}(X \times Y, X \bigvee Y)$ $\rightarrow \pi_{n}(X \vee Y)$ be the homotopy boundary operator of the pair $(X \times Y, X \bigvee Y)$. It is known [17, Theorem 18] that $i_{1}, i_{2}$, and $\partial$ define an injective representation

$$
\pi_{r}(X \vee Y) \approx \pi_{r}(X) \oplus \pi_{r}(Y) \oplus \pi_{r+1}(X \times Y, X \vee Y) \quad(r \geqq 2)
$$

Moreover, the injection $\pi_{r}(X) \rightarrow \pi_{r}(X \vee Y, Y)$ is a monomorphism.

Define a map $\phi: X * Y \rightarrow S(X \times Y)$ by

$$
\phi((1-t) x \oplus t y)= \begin{cases}2(1-t)(x, y) \oplus(2 t-1) p_{+} & (1 / 2 \leqq t \leqq 1), \\ 2 t(x, y) \oplus(1-2 t) p_{-} & (0 \leqq t \leqq 1 / 2) ;\end{cases}
$$

$\phi$ is continuous since it is well-defined. Furthermore, $\phi\left(x_{0} * Y\right) \subset S\left(x_{0} \times Y\right)$ and $\phi\left(X * y_{0}\right) \subset S\left(X \times y_{0}\right)$; hence $\phi\left(X * y_{0} \cup x_{0} * Y\right) \subset S(X \vee Y)$

LemMA 7.1. The homotopy groups of $X * y_{0} \cup x_{0} * Y$ vanish in all dimensions.

Proof. The sets $X * y_{0}$ and $x_{0} * Y$, as well as their intersection $x_{0} * y_{0}$, are contractible. Since $x_{0}$ is a strong neighborhood deformation retract of $X$, it follows that $x_{0} * y_{0}$ is a strong neighborhood deformation retract of $X * y_{0}$. Our result now follows from (3.2).

The map $\phi$, together with the inclusion map $S(X \vee Y) \subset S(X \times Y)$, defines a map $\Phi: S(X \bigvee Y) \bigvee(X * Y) \rightarrow S(X \times Y)$.

LEMMA 7.2. The homomorphism

$$
\Phi_{*}: \pi_{r}(S(X \vee Y) \vee(X * Y)) \rightarrow \pi_{r}(S(X \times Y))
$$

is an isomorphism for all $r$. 
Proof. Since both spaces are 1-connected, it suffices, by an argument of J. H. C. Whitehead $[19, \S 6]$, to show that $\Phi$ induces isomorphisms of the homology groups. Now $\Phi$ maps the homology sequence of the pair $(S(X \vee Y)$ $\vee(X * Y), S(X \vee Y))$ into the homology sequence of the pair $(S(X \times Y$, $S(X \vee Y))$, and induces the identity map on the homology groups of $S(X \vee Y)$. By the five-lemma, it suffices to show that $\Phi$ maps the relative homology groups isomorphically. However, the triad $(S(X \vee Y) \vee(X * Y) ; S(X \vee Y)$, $X * Y))$ is proper; hence the reduced homology groups of $X * Y$ are mapped isomorphically by the injection onto the homology groups of $(S(X \vee Y)$ $\vee(X * Y), S(X \vee Y))$; the former groups are isomorphic, under the injection, with the homology groups of $\left(X * Y ; X * y_{0} \cup x_{0} * Y\right)$. Hence it suffices to show that $\phi$ maps the homology groups of $\left(X * Y, X * y_{0} \cup x_{0} * Y\right)$ isomorphically onto those of $(S(X \times Y), S(X \bigvee Y))$.

Lemma 7.3. Let $f:\left(X, X_{0}\right) \rightarrow\left(Y, Y_{0}\right)$ be a relative homeomorphism [9, Chap. $\mathrm{X}]$. Suppose that $Y$ is an identification space of $X$ under $f$ and that $X_{0}$ is a strong deformation retract of a neighborhood $U$ of $X_{0}$. Then $f_{*}: H_{q}\left(X, X_{0}\right) \approx H_{q}\left(Y, Y_{0}\right)$ for all $q$.

Proof. Let $V=f(U)$. Then $V$ is a neighborhood of $Y_{0}$ and $Y_{0}$ is a strong deformation retract of $V$. Consider the commutative diagram

$$
\begin{array}{lc}
H_{q}\left(X, X_{0}\right) \stackrel{f_{1}}{\longrightarrow} & H_{q}\left(Y, Y_{0}\right) \\
\quad \downarrow i_{1} & \downarrow i_{2} \\
H_{q}(X, U) \stackrel{f_{2}}{\longrightarrow} & H_{q}(Y, V) \\
\quad \downarrow i_{3} & \downarrow i_{4} \\
H_{q}\left(X-X_{0}, U-X_{0}\right) \stackrel{f_{3}}{\rightarrow} H_{q}\left(Y-Y_{0}, V-Y_{0}\right)
\end{array}
$$

in which $i_{1}, \cdots, i_{4}$ are injections and $f_{1}, f_{2}, f_{3}$ are induced by $f$. Since $X_{0}$ and $Y_{0}$ are strong deformation retracts of $U, V$, it follows that $i_{1}$ and $i_{2}$ are isomorphisms. Since $X_{0}=\bar{X}_{0} \subset U$ and $Y_{0}=\bar{Y}_{0} \subset V$, it follows from the excision theorem for singular homology [9, Theorem VII, 9.1] that $i_{3}$ and $i_{4}$ are isomorphisms. Since $f$ maps $\left(X-X_{0}, U-X_{0}\right)$ homeomorphically onto $\left(Y-Y_{0}\right.$, $\left.V-Y_{0}\right)$ it follows that $f_{3}$ is an isomorphism. Hence $f_{1}$ is an isomorphism.

The maps

$$
\Phi:(X * Y ; X * \varnothing \cup \varnothing * Y) \rightarrow\left(S(X \times Y), P^{\prime}\right)
$$

and

$$
\Phi \mid X * y_{0} \cup x_{0} * Y:\left(X * y_{0} \cup x_{0} * Y, X * \varnothing \cup \varnothing * Y\right) \rightarrow\left(S(X \vee Y), P^{\prime}\right)
$$

satisfy the hypotheses of Lemma 7.3. Hence $\Phi$ maps the homology groups of $(X * Y, X * \varnothing \cup \varnothing * Y)$ and of $\left(X * y_{0} \cup x_{0} * Y, X * \varnothing \cup \varnothing * Y\right)$ isomorphically onto those of $\left(S(X \times Y), P^{\prime}\right)$ and $\left(S(X \vee Y), P^{\prime}\right)$. The desired conclusion now follows from the five-lemma. 
COROLlaRY 7.4. The homomorphism $\phi_{*}: \pi_{r}(X * Y) \rightarrow \pi_{r}(S(X \times Y), S(X \bigvee Y))$ is a monomorphism for all $r$. If $X$ is $(m-1)$-connected and $Y$ is $(n-1)$-connected, then $\phi_{*}$ is an isomorphism for $r \leqq m+n+\min (m, n)$.

Proof. Consider the commutative diagram

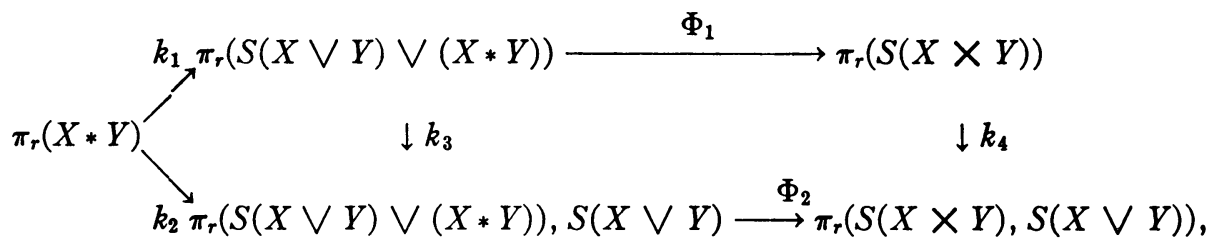

the homomorphisms $k_{i}$ are injections, while the $\Phi_{i}$ are induced by $\Phi$. From the properties of the homotopy groups of the union of two spaces with a point in common which were noted above, $k_{1}, k_{2}$, and $k_{3}$ are monomorphisms; hence $k_{4}$ is also a monomorphism. This proves the first statement. As to the second, note that $X * Y$ is $(m+n)$-connected and $S(X \vee Y)$ is $(\min (m, n))$ connected; hence [3, Theorem I] the triad $(S(X \vee Y) \vee(X * Y) ; X * Y$, $S(X \vee Y))$ is $(m+n+\min (m, n))$-connected. From the exactness of the homotopy sequence of this triad, we conclude that $k_{2}$ is onto, and therefore an isomorphism for $r \leqq m+n+\min (m, n)$.

We now define a homomorphism $\sigma: \pi_{r}(X \times Y, X \bigvee Y) \rightarrow \pi_{r+1}(X * Y)$. Let $E$ be an oriented $r$-cell. Then $E \times I$ is an oriented $(r+1)$-cell (the orientation being the cross-product of the given orientation of $E$ with the natural orientation of $I)$. Let $g:(E, \dot{E}) \rightarrow(X \times Y, X \vee Y)$ be a map; we have $g(u)=\left(g_{1}(u)\right.$, $\left.g_{2}(u)\right)$ for $u \in E$, where $g_{1}: E \rightarrow X$ and $g_{2}: E \rightarrow Y$ are maps. Define a map $\sigma g$ of $E \times I$ into $X * Y$ by

$$
\sigma g(u, t)=(1-t) g_{1}(u) \oplus \operatorname{tg}_{2}(u) \quad(u \in E, t \in I) ;
$$

then $\sigma g:\left(E \times I,(E \times I)^{\cdot}\right) \rightarrow\left(X * Y, X * y_{0} \cup x_{0} * Y\right)$, and it is easy to see that the map $g \rightarrow \sigma g$ induces a homomorphism $\sigma_{0}: \pi_{r}(X \times Y, X \vee Y) \rightarrow \pi_{r+1}(X * Y$, $\left.X * y_{0} \cup x_{0} * Y\right)$. We define $\sigma$ to be the composition with $\sigma_{0}$ of the inverse of the injection of $\pi_{r}(X * Y)$ into $\pi_{r}\left(X * Y, X * y_{0} \cup x_{0} * Y\right)$; this injection is an isomorphism because of Lemma 7.1.

LEMмA 7.5. The composition $\phi_{*} \circ \sigma: \pi_{r}(X \times Y, X \bigvee Y) \rightarrow \pi_{r+1}(S(X \times Y)$, $S(X \vee Y))$ is the (relative) Freudenthal suspension $E$.

Proof. We have

$$
\begin{aligned}
\phi(\sigma g(u, t)) & =\phi\left((1-t) g_{1}(u) \oplus t g_{2}(u)\right) \\
& = \begin{cases}2 \operatorname{tg}(u) \oplus(1-2 t) p_{-} & (0 \leqq t \leqq 1 / 2), \\
2(1-t) g(u) \oplus(2 t-1) p_{+} & (1 / 2 \leqq t \leqq 1) .\end{cases}
\end{aligned}
$$

This is clearly the suspension of the map $g$. 
LeмmA 7.6. Let $(Z, C)$ be a pair, and suppose that $(Z, C)$ is s-connected $(s \geqq 2), C$ is $t$-connected $(1 \leqq t<s)$; the suspension $E: \pi_{r}(Z, C) \rightarrow \pi_{r+1}(S Z, S C)$ is an isomorphism for $r \leqq s+t$ and an epimorphism for $r=\cdot s+t+1$.

Proof. Consider the commutative diagram

$$
\begin{array}{ccc}
\pi_{r}(Z, C) \stackrel{i_{1}}{\longrightarrow} \pi_{r}(Z \div C, \widehat{C}) & \stackrel{i_{2}}{\longleftarrow} \pi_{r}(Z \div C) \\
\downarrow E & \downarrow E_{1} & \downarrow E_{2} \\
\pi_{r+1}(S Z ; S C) \underset{i_{3}}{\longrightarrow} \pi_{r+1}(S(Z \div C), S \widehat{C}) \underset{i_{4}}{\longrightarrow} \pi_{r+1}(S Z \div S C)
\end{array}
$$

where $i_{1}, i_{2}, i_{3}, i_{4}$ are injections (note that $i_{2}$ and $i_{4}$ are isomorphisms), and $E, E_{1}, E_{2}$ are Freudenthal suspensions. By [14, Corollary 3.3], $i_{1}$ is an isomorphism for $r \leqq s+t$ and is onto for $r=s+t+1$. Since $(S Z, S C)$ is $(s+1)$ connected and $S C$ is $(t+1)$-connected, we see in the same way that $i_{3}$ is an isomorphism for $r \leqq s+t+2$. On the other hand, $Z \div C$ is $s$-connected and hence $E_{2}$ is an isomorphism for $r \leqq 2 s$.

CoRollaRy 7.7. If $X$ is $(m-1)$-connected and $Y$ is $(n-1)$-connected, then $\sigma: \pi_{r}(X \times Y, X \vee Y) \rightarrow \pi_{r+1}(X * Y)$ is an isomorphism for $r \leqq m+n+\min (m, n)$ -2 and an epimorphism for $r=m+n+\min (m, n)-1$.

Proof. In view of Corollary 7.4 and Lemma 7.5, it suffices to observe that $E: \pi_{r}(X \times Y, X \vee Y) \rightarrow \pi_{r+1}(S(X \times Y), S(X \vee Y))$ has the desired properties. Now $(X \times Y, X \vee Y)$ is $(m+n-1)$-connected [10, Theorem 2.1] and $X \vee Y$ is $t$-connected $(t=\min (m, n)-1)$; by Lemma $7.6, E$ is an isomorphism for $r \leqq m+n+t-2$ and an epimorphism for $r=m+n+t-1$.

We can now translate the results of $\$ 5$ to give some information about the homotopy groups of $(X \times Y, X \vee Y)$.

THEOREM 7.8. Let $X$ be an $(m-1)$-connected space, $Y$ an $(n-1)$-connected $C W$-complex $(m, n>1)$. Then there is a spectral sequence $\left\{E^{r}\right\}$ with the following properties:

(1) $E_{p, q}^{2}=H_{n+p}\left(Y: \pi_{m+q}(X)\right)$

(2) Let $\pi_{p, q}$ be the image of the injection

$$
(q \leqq m-2)
$$

$$
\pi_{m+n+p+q}\left(X \times Y^{n+p}, X \vee Y^{n+p}\right) \rightarrow \pi_{m+n+p+q}(X \times Y, X \vee Y) ;
$$

then, for $p+q \leqq \min (m, n)-2$, we have

$$
E_{p, q}^{\infty} \approx \pi_{p, q} / \pi_{p-1, q+1}
$$

Corollary 7.9. If furthermore $X=K(G, m)$, then

$$
\pi_{m+n+s}(X \times Y, X \vee Y) \approx H_{n+s}(Y ; G)
$$

for $s \leqq \min (m, n)-2$. 
Suppose that $X=K(G, m) Y=K(\Pi, n), m \leqq n$. Then $\pi_{m+n+s}(X \times Y, X \bigvee Y)$ $\approx H_{n+s}(\Pi, n ; G)$ for $s \leqq m-2$. Thus this group is in general not zero. On the other hand, the "generalized Whitehead products" $\alpha \star \beta \in \pi_{m+n+p+q}(X \times Y$, $X \bigvee Y),\left(\alpha \in \pi_{m+q}(X), \beta \in \pi_{n+p}(Y)\right)[2, \S 5]$, are all zero for $p+q>0$. This shows that the groups $\pi_{m+n+s}(X \times Y, X \bigvee Y)$, even in low dimensions, are not generated by generalized Whitehead products. A different example to illustrate this phenomenon has been given by Hilton [10].

\section{BIBLIOGRAPHY}

0. M. G. Barratt and J. H. C. Whitehead, On the exact couple of a CW-triad, Proc. London Math. Soc. (3) vol. 5 (1955) pp. 330-341.

1. A. L. Blakers, Some relations between homology and homotopy groups, Ann. of Math. vol. 49 (1948) pp. $428-461$.

2. A. L. Blakers and W. S. Massey, Products in homotopy theory, Ann. of Math. vol. 58 (1953) pp. 295-324.

3. - The homotopy groups of a triad II, Ann. of Math. vol. 55 (1952) pp. 192-201.

4. H. Cartan, Algèbres d'Eilenberg-MacLane et homotopie, Paris, 1955. 231-251.

5. S. Eilenberg, Cohomology and continuous mappings, Ann. of Math. vol. 41 (1940) pp.

6. Continuous mappings of infinite polyhedra, Ann. of Math. vol. 42 (1941) pp. $459-468$.

7. S. Eilenberg and S. MacLane, Relations between homology and homotopy groups of spaces, Ann. of Math. vol. 46 (1945) pp. 480-509.

8. - On the groups $H(\pi, n)$, I, Ann. of Math. vol. 58 (1953) pp. 55-106.

9. S. Eilenberg and N. E. Steenrod, Foundations of algebraic topology, Princeton University Press, 1952.

10. P. J. Hilton, On the homotopy groups of unions of spaces, Comment. Math. Helv. vol. 29 (1955) pp. 59-92.

11. W. S. Massey, Homotopy groups of triads, Proceedings of the International Congress of Mathematicians, Cambridge, Mass., 1950, Providence, American Mathematical Society, 1952, vol. 2, pp. 371-382.

12. - Exact couples in algebraic topology, Ann. of Math. vol. 56 (1952) pp. 363-396 and vol. 57 (1953) pp. 248-286. 430-436.

13. J. W. Milnor, Construction of universal bundles. II, Ann. of Math. vol. 63 (1956) pp.

14. J. C. Moore, Some applications of homology theory to homotopy problems, Ann. of Math. vol. 58 (1953) pp. 325-350.

15. - On homotopy groups of spaces with a single non-vanishing homotopy group, Ann. of Math. vol. 59 (1954) pp. 549-557.

16. F. Peterson, Generalized cokomology groups, Amer. J. Math. vol. 78 (1956) pp. 259-281.

17. G. W. Whitehead, A generalization of the Hopf invariant, Ann. of Math. vol. 51 (1950) pp. $192-237$.

18. J. H. C. Whitehead, Note on a theorem due to Borsuk, Bull. Amer. Math. Soc. vol. 54 (1948) pp. 1125-1132.

19. - Combinatorial homotopy I, Bull. Amer. Math. Soc. vol. 55 (1949) pp. 213-245.

Massachusetts Institute of Technology,

Cambridge, Mass. 